\title{
Delteil en détail, sous la direction de Anne-Lise Blanc
}

\section{Michela Colombo}

\section{(2) OpenEdition}

\section{Journals}

\section{Edizione digitale}

URL: http://journals.openedition.org/studifrancesi/3852

DOI: $10.4000 /$ studifrancesi.3852

ISSN: 2421-5856

\section{Editore}

Rosenberg \& Sellier

\section{Edizione cartacea}

Data di pubblicazione: 1 décembre 2012

Paginazione: 608

ISSN: 0039-2944

\section{Notizia bibliografica digitale}

Michela Colombo, «Delteil en détail, sous la direction de Anne-Lise Blanc», Studi Francesi [Online], 168 (LVI | III) | 2012, online dal 30 novembre 2015, consultato il 07 mars 2021. URL: http:// journals.openedition.org/studifrancesi/3852 ; DOI: https://doi.org/10.4000/studifrancesi.3852

Questo documento è stato generato automaticamente il 7 mars 2021.

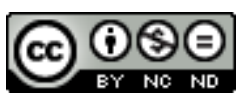

Studi Francesi è distribuita con Licenza Creative Commons Attribuzione - Non commerciale - Non opere derivate 4.0 Internazionale. 


\title{
Delteil en détail, sous la direction de Anne-Lise Blanc
}

\author{
Michela Colombo
}

\section{NOTIZIA}

Delteil en détail, sous la direction de Anne-Lise BLANC, Perpignan, Presses Universitaires de Perpignan, 2011, pp. 191.

1 Il presente volume raccoglie i contributi di studiosi dalle personalità estremamente eterogenee, accomunati da un vivo interesse per l'opera di Joseph Delteil. Alcuni di essi hanno partecipato alla giornata di studio intitolata Delteil en détail organizzata all'Université de Perpignan nel marzo 2010, altri invece hanno offerto successivamente il loro contributo aggiungendo le loro voci al progetto iniziale. Tutti gli studi qui riuniti contribuiscono a tracciare un ritratto letterario di Joseph Delteil mettendone in evidenza al contempo le molteplici sfaccettature e la pluralità di profili e interessandosi tanto alle sue fonti di ispirazione quanto alla sua fervida creatività. Viene in effetti a emergere l'immagine di un autore oggi ancora poco studiato che non temeva di oltrepassare troppo angusti confini di genere per mescolarli in modi sempre originali e innovativi, e la varietà caratteristica della sua opera unita a una innegabile potenza poetica sembrano alimentarne la singolare e certamente interessante estetica. La raccolta si propone pertanto di esplorare l'opera di Delteil, di evidenziarne la ricchezza e la diversità dei generi, dei toni e delle posizioni. In particolare, sarà il gioco costante a cui l'autore sottopone le frontiere di genere ad essere esaminato nei contributi di Marie-Françoise LEMONNIER-DELPY ("Je hais comme la peste les classifications et les genres", pp.15-34), di Cécile YaPaudijan (La poésie des noms dans "Choléra" de Joseph Delteil, pp.35-50) e di Anne-Lise BlANC ("Perpignan" ou la matière romanesque d'un paysage sensible, pp. 51-66). Un'analisi dei modelli di riferimento a cui Delteil si rifà nel corso della sua produzione, quali forme classiche, racconti di vite, leggende e personaggi, è invece al centro dei contributi di Mireille COURRENT (Delteil avant Delteil: les mouvements 
du marbre dans "Le Cour grec", pp. 67-84), Aude BONORD (Joseph Delteil, inventeur de vies, pp. 85-102), Rim TAGA GABSI (Delteil tel qu'on l'ignore: chantre de Jeanne d'Arc, pp. 103-118) e Mathieu GIMENEZ (Les Petits plats de Joseph Delteil: un retour... vers le futur?, pp. 119-138). Un interesse maggiormente volto verso l'uomo, o meglio, verso il personaggio Delteil, è infine al centro dei testi di Robert BRIATTE ( $A u$ nom du frère, du petit père des peuples et $d u$ libre esprit. Les trois Joseph, pp. 139-154) e di Hyacinthe CARRERA (Joseph Delteil, paysan? Une écriture de cueillette plutôt que de culture, pp. 155-176), i quali offrono una disamina delle immagini che l'autore ha lasciato di se stesso, per tentare, se non di situare, almeno di seguire Delteil fra i diversi ambiti e i diversi movimenti del secolo.

2 A tali contributi di ordine accademico si aggiunge infine quello di un'artista, Colette NYS-MAZURE - Joseph Delteil. Lectures croisées ("Célébration du quotidien", "Singulières et plurielles" et "Feux dans la nuit"), fécondité d'une cuvre -, scrittrice per la quale la lettura di Delteil è stata determinante: sfruttandone la contagiosa energia generativa, ella propone una lettura incrociata dell'opera dell'autore con quella di altri scrittori quali Rabelais, Colette, Norge, Albert Camus, René Char e Marguerite Yourcenar.

3 In ultimo, occorre segnalare le illustrazioni create appositamente per il volume da Gérard BLANc, le quali coadiuvano lo spirito dell'opera declinando, sulla scia di alcuni modelli (i cavalli di Delacroix, le fotografie originali di Joseph Delteil), ma in maniera mirabilmente originale, la natura di un personaggio dalle molteplici e sorprendenti sfaccettature. 